\title{
Implementierung eines schräg angeströmten Rechens in die 3D-numerische Berechnung mit FLOW-3D
}

\author{
Sebastian Krzyzagorski · Roman Gabl · Jakob Seibl · Heidi Böttcher · Markus Aufleger \\ Online publiziert: 17. Februar 2016 \\ C Die Autor(en) 2016. Dieser Artikel ist auf Springerlink.com mit Open Access verfügbar.
}

Zusammenfassung In den letzten Jahren hielt vermehrt die dreidimensionale (3D-)numerische Strömungssimulation im Bereich der Grundlagenforschung und der Planung von hydraulischen Anlagen Einzug. Rechen im Bereich von Kraftwerkseinläufen stellen jedoch für die numerische Simulation eine besondere Herausforderung dar. Dies ist der, im Vergleich zu anderen Bauteilen, kleinteiligen Rechenstabgeometrie geschuldet. Als alternativer Lösungsansatz zur Modellierung der Rechenverluste in der Software FLOW-3D bietet sich als Flächenobjekt eine Baffle an. Der Beitrag baut auf den Erkenntnissen von Waldy et al. (Österreichische Wasser- und Abfallwirtschaft 67:1-2, 2015) auf, welcher unterschiedliche Konzepte zur vereinfachten Modellierung der Verluste an Rechen untersucht hat. Dabei zeigt sich, dass die Verwendung einer Baffle als erfolgversprechende Variante gesehen werden kann. Diese Vergleiche erfolgten jedoch unter der Einschränkung eines gerade angeströmten Rechens, bei dem die Netzausrichtung immer in der Rechenebene lag. Der aktuelle Beitrag befasst sich mit der Modellierung der Rechenverluste mithilfe einer Baffle am schräg gestellten Rechen und erweitert somit den Beitrag Waldy et al. (Österreichische Wasser- und Abfallwirtschaft 67:1-2, 2015).

\footnotetext{
Dr. R. Gabl $(\bowtie) \cdot$ DI S. Krzyzagorski • DI J. Seibl · DI H. Böttcher . Univ.-Prof. Dr.-Ing. M. Aufleger Arbeitsbereich Wasserbau, Universität Innsbruck, Technikerstraße 13, 6020 Innsbruck, Österreich E-Mail: roman.gabl@uibk.ac.at
}

Implementation of an angled trash rack in the 3D-numerical simulation with FLOW-3D

Summary Over the last years, scientists and engineers have used more and more 3D-numerical flow simulations for basic research and the planning of hydraulic constructions. However, trash racks in front of the intakes of hydroelectric power plants represent a particular problem for numerical simulations. The reason for this is the especially fragmented geometry of the trash rack bars in comparison to other construction elements. As an alternative approach to include trash rack losses into a 3Dnumerical simulation with FLOW-3D a baffle can be used. Waldy et al. (Österreichische Wasser- und Abfallwirtschaft $67: 1-2,2015)$ demonstrated that such a baffle is a promising method to model the losses at trash racks. These comparisons of different concepts were limited to a vertical trash rack, which had its grid orientation along the computational plane. The actual paper focuses on the modelling of the trash rack losses by means of a baffle at an angled trash rack and thus upgrades the survey of Waldy et al. (Österreichische Wasser- und Abfallwirtschaft $67: 1-2,2015$ ).

\section{Einleitung}

\subsection{Problemstellung}

Um die durch den Rechen verursachten Energieverlustebeieiner3D-numerischen Simulation $\mathrm{zu}$ berücksichtigen, ist ein möglicher Ansatz, die Geometrie der einzelnen Rechenstäbe direkt zu berücksichtigen. Dazu bedarf es einer sehr feinen Netzdichte, um überhaupt die Geometrie korrekt zu diskretisieren. Zudem ist noch eine weitere Verfeinerung des Netzes erforderlich, um eine korrekte Berechnung der Strömung sicherzu- stellen (Gabl et al. 2014; Waldy et al. 2015). Eine erhöhte Anzahl an Rechenzellen bedeutet eine längere Rechenzeit, was aus wirtschaftlichen Gründen sehr schnell nicht mehr vertretbar ist. Die Entwicklungen im Bereich des Fischschutzes führen tendenziell vermehrt zu kleinen lichten Weiten zwischen den einzelnen Rechenstäben. Diese werden abhängig von der zu schützenden Fischart bemessen und können $20 \mathrm{~mm}$ unterschreiten (Cuchet 2013; Rettemeier 2000). Durch die geringen Stababmessungen und lichten Weiten, die sich aus den Anforderungen des Fischschutzes ergeben, verschärft sich diese Herausforderung bei der Implementierung in die 3D-numerische Berechnung noch weiter. Waldy et al. (2015) untersuchten, wie die Rechenverluste mit der 3D-numerischen Simulationssoftware FLOW-3D unter Zuhilfenahme alternativer Lösungsansätze modelliert werden können. Ziel dieser Untersuchungen war es, die notwendige Netzdichte zu reduzieren und trotzdem noch vergleichbare Ergebnisse zu erhalten. Dabei zeigte sich, dass sich sogenannte Baffles als Variante zur Modellierung der lokalen Rechenverluste anbieten, da die modellierten Verlusthöhen des Rechens nicht von der Netzdichte abhängen. Diese Untersuchungen erfolgten nur für gerade angeströmte Rechen und einer Baffle, die immer am Übergang zweier Netzelemente angeordnet war. Damit erfolgte die Ausrichtung der Baffle ebenfalls am orthogonalen Berechnungsnetz. Der Grund liegt in der Definition dieser Flächenobjekte selbst, welche in FLOW-3D in ihren Eigenschaften mehr einer Oberfläche denn einem Volumenobjekt ähneln (Flow Science 2014). Dementsprechend werden sie nicht als Zellen abgebildet, sondern nur zwischen den Zellen eines Netzes. Damit wird eine zwar frei wählbare Eingabe für die Berechnung von der Software selbst trotzdem immer 
zu den Gitterlinien des Netzes orientiert. Bei einer schräg zum Netz ausgerichteten Baffle ergibt dies eine abgestufte Oberflächendefinition. Im Rahmen dieses Beitrags werden die Auswirkungen einer Baffle untersucht, welche nicht an der Berechnungsebene ausgerichtet ist und somit von der Software deformiert wird. Ziel ist es zu untersuchen, ob die Simulation der Verluste eines Rechens mit dem vereinfachten Lösungsansatz der Baffle auch dann angewendet werden kann, wenn der Rechen schräg angeströmt wird und die Baffle nicht in Berechnungsebene ausgerichtet ist.

\subsection{Theoretische Grundlagen}

AlsFormelfürdieBerechnungderEnergieverluste am Rechen wurde der Ansatz von Meusburger (2002) gewählt. Dieser baut teilweise auf den Erkenntnissen von Kirschmer (1926) auf. Kirschmer (1926) konzentrierte sich in seiner Arbeit auf die Untersuchungen am gerade eingebauten und vertikal geneigten Rechen, und zwar jeweils mit vertikal orientierten Rechenstäben und ohne Einfluss von Verlegung durch Treibgut. Je größer dabei die Schrägstellung des Rechens bezogen auf die Vertikale ist, desto größer wird die Fläche, die dem Wasser zum Durchfluss zur Verfügung steht. Die vertikale und die schräge Länge werden über die Sinusfunktion der Rechenneigung $\alpha$ miteinander verbunden. Dabei ist $\alpha$ als der Winkel zwischen der Horizontalen und dem geneigten Rechen definiert. Würde das Wasser immer senkrecht auf die geneigte Rechenfläche strömen, würde die Geschwindigkeit mit dem Wert $\sin (\alpha)$ abnehmen und der Rechenverlust würde somit um $\sin ^{2}(\alpha)$ reduziert werden. Kirschmer (1926) zeigte aber, dass der Verlust nur mit dem Wert $\sin (\alpha)$ zurückgeht. Meusburger (2002) leitete in seiner Arbeit anhand von Modellversuchen und zahlreichen Erhebungen in der Praxis an bestehenden Kraftwerksbauten eine neue Formel auf empirischer Basis her, die eine praxistaugliche Abschätzung der Energieverluste am Einlaufrechen ermöglichen soll. Als Basis für seine Formel verwendet er den Stabformbeiwert $\mathrm{k}_{\mathrm{F}}$ und den Faktor $\sin (\alpha)$ für die Rechenneigung nach Kirschmer (1926). Meusburger (2002) stellte die folgende Gleichung zur Berechnung der Energieverlusthöhe $\Delta \mathrm{h}_{\mathrm{E}}$ am Rechen auf:

$$
\Delta h_{E}=\zeta_{R} * \frac{v_{R}^{2}}{2^{*} g}
$$

Die Geschwindigkeit $v_{R}$ bezieht sich dabei auf den unverbauten Querschnitt vor dem Rechen und $g$ ist die Erdbeschleunigung. Der Verlustbeiwert $\zeta_{R}$ ist dabei folgendermaßen definiert:

$$
\zeta_{R}=\zeta_{P} * k_{\delta} * k_{V} * k_{\alpha}
$$

Die Teilfaktoren für die Gl. 2 sind wie folgt definiert:

\begin{tabular}{|c|l|}
\hline$\zeta_{p}$ & Verlustbeiwert infolge Verbauung \\
\hline $\mathrm{k}$ & $\begin{array}{l}\text { Anpassung infolge horizontaler } \\
\text { Schräganströmung mit dem Winkel } \delta\end{array}$ \\
\hline $\mathrm{k}_{\mathrm{v}}$ & $\begin{array}{l}\text { Faktor für die Berücksichtigung einer } \\
\text { Rechenverlegung }\end{array}$ \\
\hline $\mathrm{k}$ & $\begin{array}{l}\text { Anpassung entsprechend einer } \\
\text { vertikalen Rechenneigung mit dem } \\
\text { Winkel } \alpha\end{array}$ \\
\hline
\end{tabular}

Für die detaillierte Bestimmung wird auf Meusburger (2002) verwiesen.

Als Basis für die Konfigurationen des Rechens, welche in weiterer Folge untersucht werden, dient ein laufendes Forschungsprojekt am Arbeitsbereich (AB) Wasserbau der Universität Innsbruck (Aufleger 2014). Ritsch (2015) untersucht im Zuge seiner Masterarbeit die Verlusthöhen des Rechens bei unterschiedlichen Rechenkonfigurationen anhand eines Vorversuchs zum Seilrechen.

Infolge der Anforderungen an einen fischfreundlichen Rechen ergeben sich bei klassischen Rechenkonstruktionen Herausforderungen durch die erhöhten hydraulischen Verluste, die hohen Errichtungskosten und den aufwendigen Betrieb (Böttcher et al. 2014; Aufleger und Brinkmeier 2015). Deshalb wird seit 2012 am AB Wasserbau der Seilrechen als neues Fischschutzsystem entwickelt und erprobt. Dieser besteht aus horizontalen Seilen, die schräg über den gesamten Einlaufquerschnitt gespannt sind. Der Seilrechen bietet eine zum Rechen mit vertikalen Rechenstäben, die aus der Lotrechten geneigt sind, vergleichbare Einbausituation. Meusburger (2002) benennt in seinem Formelwerk den vertikalen Winkel mit $\alpha$ und den horizontalen Winkel mit $\delta$. Zum besseren Verständnis sind die beiden Rechenkonfigurationen samt Winkelbezeichnungen in Abb. 1 dargestellt. Der blaue Pfeil markiert die Fließrichtung. Die Rechenstäbe werden bei beiden Bauarten quer zu ihrer Längs-

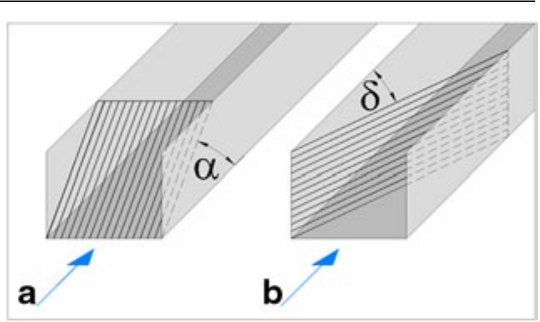

Abb. 1 a Vertikal geneigte Rechenstäbe mit Winkel $\alpha$ nach Definition von Meusburger (2002) und b Seilrechen mit Winkel $\delta$

achse geneigt. Aufgrund dessen kann näherungsweise der horizontale Anströmwinkel $\delta$ (Abb. 1b) dem Winkel der vertikalen Rechenneigung $\alpha$ von Meusburger (2002) in Abb. la gleichgesetzt werden. Mit der horizontalen Neigung des Seilrechens verändert sich damit der Teilfaktor $\mathbf{k}_{\alpha}$ nach Meusburger (2002). Dieser ist mit $k_{\alpha}=\sin (\alpha)$ definiert und wird hier $\mathrm{zu} \mathrm{k}_{\alpha}=\sin (\delta)$ umgeformt. Der vertikale Neigungswinkel der Ebene, in welcher die Seile gespannt werden, wird im Rahmen dieser Arbeit nicht verändert, wodurch der Seilrechen als konstant senkrecht vereinfacht wird. Damit resultiert der Beiwert $\mathrm{zu} \mathrm{k}_{\delta}=1,0$ [-]. Zudem liegt vergleichbar zu Waldy et al. (2015) der Fokus rein auf der gesamthaften Betrachtung der Verlusthöhe und nicht auf der direkten Strömungsbeeinflussung durch ein einzelnes Seil.

\subsection{Baffles und Implementierung der Verlusthöhe in FLOW-3D}

Baffles sind Objekte mit den Eigenschaften einer Fläche und dienen ursprünglich in der numerischen Simulation von Strömungsvorgängen aus dem Bereich der Gießtechnik als lokale simple Sperren von Gießkanälen. Definiert sind sie als Ebenen, als Zylinder oder als komplexe Flächen, deren Form einer quadratischen Funktion folgt. Weil sie in ihren Eigenschaften einem Flächenobjekt entsprechen, folgt ihre Geometrie den Gitterlinien des Berechnungsnetzes. Baffles, die zwischen den Gitterlinien des Berechnungsnetzes liegen, werden von der Software automatisch zur nächsten Gitterlinie verschoben. Gekrümmte oder schräge Baffles erhalten eine treppenartige Definition. FLOW-3D erlaubt es auch, den Verlustbeiwert beim Durchströmen des Wassers durch die Ebene so einzustellen, dass eine Baffle keine Verluste erzeugt. Dann kann die Baffle auch unter anderem als Messquerschnitt 
benutzt werden um Strömungsdaten wie zum Beispiel den Durchfluss zu messen (Flow Science 2014).

Um die Verlusthöhe einer Baffle zu modellieren, bietet FLOW-3D unter dem Punkt POROSITY PROPERTIES mehrere Möglichkeiten an (Flow Science 2014):

- Porosität P,

- Linearer Verlustkoeffizient $\zeta_{\mathrm{L}}$ und

- Quadratischer Verlustkoeffizient $\zeta_{Q}$.

Der Wert für die Porosität $\mathrm{P}$ ist regelbar im Bereich von 0 (undurchlässig) bis 1 (vollkommen offen). Diese Modellierung der Verlusthöhen hat den großen Nachteil, dass der Koeffizient für die Porosität $P$ zuerst für jede Netzauflösung einzeln kalibriert werden muss. Zudem zeigt das Ergebnis eine deutliche Abhängigkeit von der Netzgröße (Waldy et al. 2015). Deshalb bietet sich die direkte Eingabe der Verlustkoeffizienten an. In FLOW-3D gehen die linearen und quadratischen Koeffizienten entsprechend der folgenden Gleichung in die Definition des Druckverlustes $\Delta \mathrm{p}$ ein (Flow Science 2014):

$$
\Delta p=\rho *\left(\zeta_{L} * v+0.5 * \zeta_{Q} * v^{2}\right)
$$

Die Geschwindigkeit v bezieht sich dabei auf jene beim Passieren der Baffle. Diese Geschwindigkeit steht in folgendem Verhältnis zur Geschwindigkeit $\mathrm{v}_{\mathrm{R}}$ im unverbauten Querschnitt (Flow Science 2014):

$$
v=\frac{v_{R}}{P}
$$

Waldy et al. (2015) modifizierten die allgemeine implementierte Gl. 4, um den Einlaufverlust mit der Formel nach Meusburger (2002) zu berechnen. Da die Verlusthöhe eine quadratische Abhängigkeit von der Geschwindigkeit besitzt, wird der lineare Verlustkoeffizient $\zeta_{\mathrm{L}}=0$ gesetzt. Die Porosität wird auf $P=1$ gesetzt, damit entspricht zufolge Gl. 4 die Geschwindigkeit $v=v_{R}$. Wird die Gleichung nun durch $\rho^{*}$ g dividiert, erhält man Gl. 5:

$$
\frac{\Delta p}{\rho^{*} g}=\zeta_{Q} * \frac{v_{R}{ }^{2}}{2^{*} g}
$$

Es besteht jedoch die Problematik, dass sich die Formel von Meusburger (2002) auf die Energiehöhendifferenz zwischen oberstrom und unterhalb des Rechens bezieht, während sich der Ansatz von FLOW-3D auf die entsprechende Differenz des Wasserspiegels bezieht. Wird dies in Gl. 5 berücksichtigt, kommt man zur Gl. 6. Dabei entspricht $\mathrm{v}_{\mathrm{D}}$ der Geschwindigkeit nach dem Rechen:

$$
\zeta_{Q} * \frac{v_{R}^{2}}{2 * g}=\zeta_{R} * \frac{v_{R}^{2}}{2 * g}-\frac{v_{R}^{2}-v_{D}^{2}}{2 * g}
$$

Bei in der Praxis üblichen Rechenanlagen ist die Einlaufgeschwindigkeit relativ gering, deshalb ist der Anteil der Geschwindigkeitshöhe vernachlässigbar klein. Wird dies berücksichtigt, gelangt man so zu Gl. 7. Wie daraus ersichtlich ist, kann $\zeta_{\mathrm{Q}}$ mit $\zeta_{\mathrm{R}}$ nach Meusburger (2002) gleichgesetzt werden (Waldy et al. 2015):

$$
\zeta_{Q} * \frac{v_{R}{ }^{2}}{2 * g}=\zeta_{R} * \frac{v_{R}{ }^{2}}{2 * g}
$$

Die Werte für die Porosität und die Verlustkoeffizienten lassen sich nur global für das gesamte Berechnungsgebiet definieren. Eine getrennte Eingabe in $x$-, $\mathrm{y}-$ und z-Richtung ist somit nicht möglich. Laut Flow Science (2014) werden die Verluste aber sehr wohl in $\mathrm{x}^{-}, \mathrm{y}-\mathrm{und}$ z-Richtung in Abhängigkeit von der jeweiligen Geschwindigkeit getrennt berechnet. Als Bezugsgeschwindigkeit wird die jeweils aktuelle MICROSCOPICVELOCITY in der Baffle herangezogen, also die Geschwindigkeit der einzelnen Teilchen beim Durchströmen der Baffle. Wie in Rettemeier (2000) beschrieben, verursachen geneigte Rechen durch die keilförmige Verengung des Durchflussquerschnittes mit fortlaufender Rechenlänge eine erhöhte Fließgeschwindigkeit. Durch die von FLOW-3D implementierte Berechnung der Geschwindigkeit eines jedes einzelnen Stromes beim Passieren der Baffle sollten damit die unterschiedlichen Fließgeschwindigkeiten im Bereich der schräg gestellten Baffle mitberücksichtigt werden.

Es wird darauf hingewiesen, dass bei einem Rechen mitvertikalen Rechenstäben und horizontaler Schräganströmung stromabwärts vom Rechen Strömungsphänomene auftreten, die sich mit einer Baffle nicht simulieren lassen. Dazu zählen z. B. Strömungsumlenkungen und Strömungsschatten in Abhängigkeit von der Profilform (Chatellier 2011; Kriewitz 2015; Mosonyi 1966; Raynal et al. 2013; Waldy et al. 2015; Zimmermann 1969).

\section{Untersuchung}

\subsection{Numerik}

Die numerischen Berechnungen erfolgten mit der Software FLOW-3D, InterfaceVersion 11.0.3.6 und Solver-Version
11.0.3.05 und sind Teil der Masterarbeit Krzyzagorski (2015), welche am Arbeitsbereich Wasserbau der Universität Innsbruck verfasst wurde. Details zur Software, Qualitätssicherung und Anwendungsbeispiele können im User Manual (Flow Science 2014) oder zum Beispiel bei Gabl et al. (2014) nachgelesen werden.

\subsection{Geometrie}

Die Geometrie der nachfolgend beschriebenen numerischen Versuche orientiert sich an den Maßen des physikalischen Vorversuchs zum Seilrechen, welcher unter anderem in der Arbeit Ritsch (2015) untersucht wird. Der rechteckige Gerinnequerschnitt in der Glasrinne im Wasserbaulabor der Universität Innsbruck besitzt eine Länge von $22,00 \mathrm{~m}$ und eine Breite von $0,80 \mathrm{~m}$. Alle Versuche werden mit einem konstanten Unterwasserspiegel von $40 \mathrm{~cm}$ durchgeführt. Im Rahmen der numerischen Untersuchung werden die Anströmwinkel $\delta=30^{\circ}, 37,5^{\circ}, 45^{\circ}, 60^{\circ}$ und $75^{\circ}$ simuliert. Um eine Vergleichsmöglichkeit für den schrägen Rechen zu bekommen, wird der Fall des geraden Rechens mit $\delta=90^{\circ}$ ebenfalls betrachtet. Nachfolgend in Abb. 2 findet sich eine Darstellung der Modellgeometrie, allerdings ohne im Detail auf die Auswertepunkte einzugehen. Für eine vollständige Darstellung der Modellgeometrie inklusive der kompletten Auswertung wird auf die Arbeit Krzyzagorski (2015) verwiesen.

\subsection{Randbedingungen}

Ein Seilrechen besteht aus horizontalen Seilen, die innerhalb einer schrägen Ebene über den gesamten Einlaufquerschnitt gespannt sind (Böttcher et al. 2014). Im Vorversuch zum Seilrechen werden diese Seile durch starre, kreisrunde Stäbe ersetzt (Aufleger 2014; Ritsch 2015). Ein exemplarisches Foto dieses Details ist in Abb. 3 dargestellt. Diese Vereinfachung für die Ermittlung des Verlustkoeffizienten (welcher für die Baffle eingegeben werden muss) wird auch im numerischen Modell übernommen, da es für kreisrunde Stäbe in der Literatur bereits Untersuchungen zum Strömungsverhalten und zum Stabformbeiwert gibt (Kirschmer 1926).

Für die numerischen Untersuchungen werden die Randbedingungen der Seitenwände und der Grundfläche auf SYMMETRY gesetzt, um etwaige Einflüsse infolge von Wandreibung auszuschließen. Im Prinzip wird so nur 


\section{GRUNDRISS GERADER RECHEN}

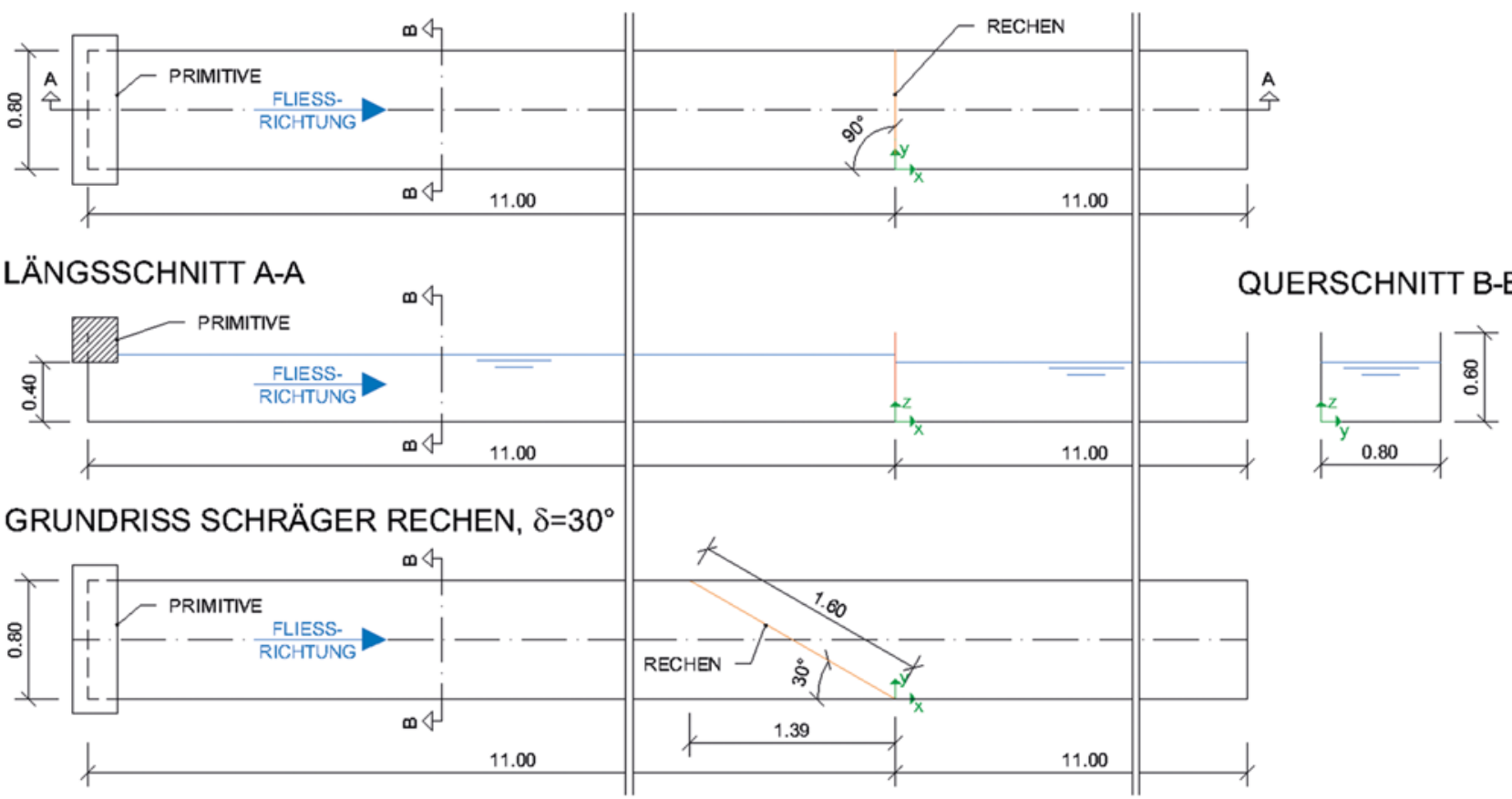

Abb. 2 Modellgeometrie, Grundriss (GR) und Schnitte für den geraden Rechen und exemplarisch der GR für den $30^{\circ}$ geneigten Rechen - Einheiten in [m]

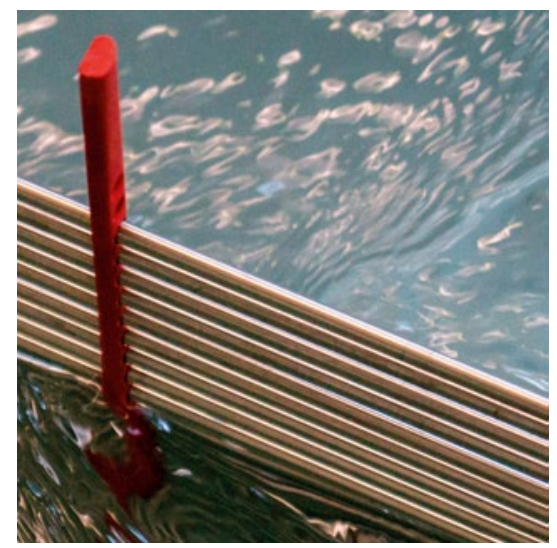

Abb. 3 Detail des Rechens am Vorversuch zum Seilrechen - Blick in Fließrichtung

ein Ausschnitt eines unendlich breiten Rechens simuliert. Als weitere Vereinfachung wird das Gerinne als vollkommen eben, also ohne Sohlgefälle, modelliert. Als obere Randbedingung $\mathrm{x}_{\min }$ kommt ein konstanter Wasserzufluss mit $v_{\mathrm{x}}=0,625 \mathrm{~m} / \mathrm{s}$ zum Einsatz, wobei die Zuströmfläche auf $40 \mathrm{~cm}$ Höhe begrenzt wird. Damit ergibt sich ein konstanter Wasserzufluss von $Q=200 \mathrm{l} / \mathrm{s}$. Als untere Randbedingung $\mathrm{x}_{\max }$ wurde ein Wasserspiegel von $40 \mathrm{~cm}$ festgelegt, dadurch ist die Randbedingung beim Einlauf immer eingestaut. Im Oberwasserbereich
Tab. 1 Verlustbeiwerte $\zeta_{\mathrm{R}}$ nach Meusburger (2002) für unterschiedliche horizontale Anströmwinkel

\begin{tabular}{|l|l|l|}
\hline $\begin{array}{l}\text { Horizontaler } \\
\text { Anströmwinkel } \\
\delta\left[^{\circ}\right]\end{array}$ & $\begin{array}{l}\text { Faktor horizontale } \\
\text { Schräganströmung } \\
\mathrm{k}_{\delta}[-]\end{array}$ & $\begin{array}{l}\text { Verlust- } \\
\text { beiwert } \\
\zeta_{R}[-]\end{array}$ \\
\hline 90 & 1,000 & 1,790 \\
\hline 75 & 0,966 & 1,729 \\
\hline 60 & 0,866 & 1,550 \\
\hline 45 & 0,707 & 1,266 \\
\hline 37,5 & 0,609 & 1,090 \\
\hline 30 & 0,500 & 0,895 \\
\hline
\end{tabular}

kommt es infolge des Rechens bzw. infolge der Baffle zu einem Einstau mit einer Wasserhöhe $>40 \mathrm{~cm}$.

Der Teilfaktor infolge Verbauung ermittelt sich mit den Eingangsdaten zu $\zeta_{\mathrm{p}}=1,79$ [-]. Eine Rechenverlegung mit Treibgut wird nicht berücksichtigt, somit bestimmt sich dieser Beiwert $k_{v}$ zu 1,0 [-]. Für die unterschiedlichen horizontalen Anströmwinkel lassen sich schlussendlich mithilfe der Gl. 2 die theoretischen Verlustbeiwerte aus Tab. 1 berechnen:

Gemäß Gl. 7 wird der theoretische Verlustbeiwert $\zeta_{\mathrm{R}}$ mit dem quadratischen Verlustkoeffizienten $\zeta_{\mathrm{Q}}$ gleichgesetzt, welcher in FLOW-3D zur Berechnung der Verluste der Baffle herangezogen wird.
Nach Kirschmer (1926) und Meusburger (2002) nimmt die Rechenverlusthöhe mit dem Faktor $\sin (\delta)$ ab. Dieser geometrische Faktor ergibt sich aus dem Verhältnis der schrägen Länge zur geraden, ungedrehten Länge des Rechens im Grundriss. Inwieweit diese Anpassung mit dem Geometriefaktor bei der Implementierung in FLOW-3D berücksichtigt werden muss, ist Teil der vorliegenden Untersuchung. Dabei stellt sich die Frage, ob für den quadratischen Verlustkoeffizienten $\zeta_{Q}$ bei geneigter Baffle der Wert $\zeta_{\mathrm{R}}$ des geneigten Rechens oder einfach nur der Wert $\zeta_{R}$ des geraden Rechens eingesetzt werden muss und FLOW-3D den Geometriefaktor selbstständig abmindert.

\subsection{Räumliche Diskretisierung}

Die räumliche Diskretisierung erfolgt bei FLOW-3D mittels eines kartesischen Koordinatensystems mit einem strukturierten, orthogonalen Netz. Alternativ bietet die Software auch ein zylindrisches Koordinatensystem zur Auswahl an (Flow Science 2014; Gabl et al. 2014). Die Zellgrößen werden für die nachfolgende Untersuchung in $\mathrm{x}-$, $\mathrm{y}$ - und z-Richtung gleich definiert, sodass die Zellen stets würfelförmig sind (Tab. 2). Die Zellgröße beim Netz N1 wird so ausgewählt, dass sich ca. 4 Zellen in der 
Tab. 2 Räumliche Diskretisierung

\begin{tabular}{|l|l|l|l|l|l|}
\hline $\begin{array}{l}\text { Netz } \\
{[\mathrm{Nr}]}\end{array}$ & $\begin{array}{l}\text { Zellgröße } \\
{[\mathrm{m}]}\end{array}$ & $\begin{array}{l}\text { Anzahl Zellen in } \\
\text { x-Richtung }\end{array}$ & $\begin{array}{l}\text { Anzahl Zellen in } \\
\mathrm{y} \text {-Richtung }\end{array}$ & $\begin{array}{l}\text { Anzahl Zellen in } \\
\text { z-Richtung }\end{array}$ & $\begin{array}{l}\text { Anzahl Zellen } \\
\text { Gesamt }\end{array}$ \\
\hline N1 & 0,1000 & 220 & 8 & 6 & 10.560 \\
\hline N2 & 0,0500 & 440 & 16 & 12 & 84.840 \\
\hline N3 & 0,0250 & 880 & 32 & 24 & 675.840 \\
\hline N4 & 0,0125 & 1760 & 64 & 48 & 5.406 .720 \\
\hline
\end{tabular}

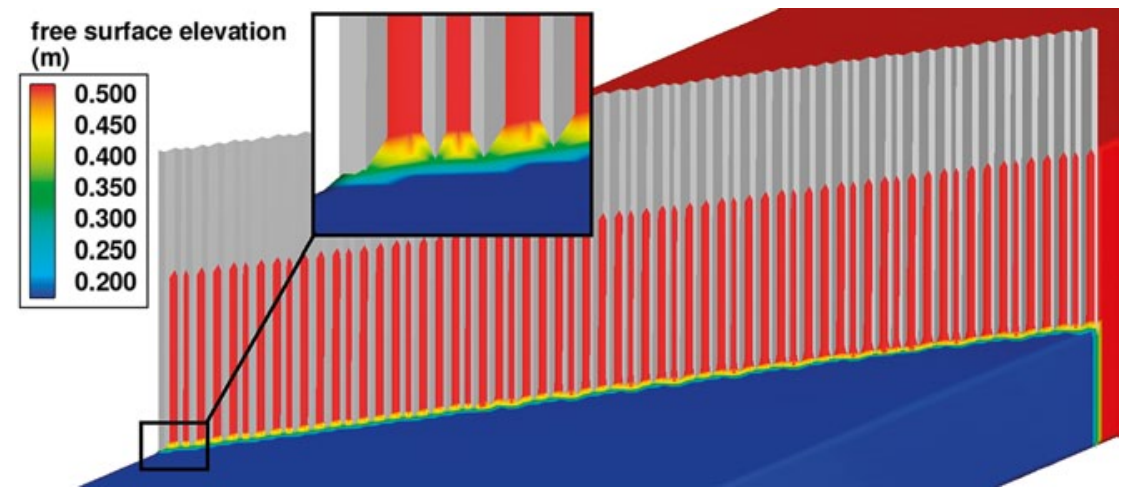

Abb. 4 3D-Ansicht der Nullvariante, geneigter Rechen, $\delta=30^{\circ}$, Netz N4

Fließhöhe ergeben, was als untere Grenze für eine stabile Berechnung gilt (Gabl et al. 2014). Mit dem jeweils nächstkleineren Netz wurde die Zellgröße immer weiter halbiert, so lange, bis mit dem Netz N4 die feinste untersuchte Netzauflösung erreicht wird.

\subsection{Methodologie der Auswertung und Ermittlung des Rechenverlustes}

Die gewählte Rechenkonfiguration der numerischen Simulation bietet in Fließrichtung gesehen sowohl vor als auch nach dem Rechen einen Freispiegelabfluss. Der Querschnitt des Gerinnes ist auf ganzer Länge konstant und ohne Sohlgefälle ausgeführt. Aufgrund der gewählten Randbedingungen können etwaige Einflüsse infolge Wandreibung minimiert werden. Unter diesen Voraussetzungen ergibt sich die Gl. 8 zur Bestimmung der Energieverlusthöhe $\Delta \mathrm{h}_{\mathrm{E}}$ (Meusburger 2002). Die Wasserspiegelhöhe im unverbauten Querschnitt vor dem Rechen wird mit $h_{R}$ und die Wasserspiegelhöhe im unverbauten Querschnitt nach dem Rechen mit $h_{D}$ bezeichnet. Aus diesen Werten können anschließend mithilfe der Kontinuitätsgleichung die Fließgeschwindigkeiten $\mathrm{v}_{\mathrm{R}}$ im unverbauten Querschnitt vor dem Rechen und $v_{D}$ im unverbauten Querschnitt nach dem Rechen berechnet werden.

$$
\Delta h_{E}=h_{R}-h_{D}+\frac{v_{R}{ }^{2}-v_{D}{ }^{2}}{2^{*} g}
$$

Für die Ermittlung der Energieverlusthöhe $\Delta \mathrm{h}_{\mathrm{F}}$ ist deshalb eine exakte Wasserspiegellage entscheidend. Um die Genauigkeit zu steigern und trotzdem akzeptable Berechnungszeiten zu bekommen, wird für jeden Messpunkt ein Mittelwert über den Zeitraum $\mathrm{t}=70-100 \mathrm{~s}$ gebildet. Für die Berechnung der Energieverlusthöhe $\Delta \mathrm{h}_{\mathrm{E}}$ sind die Messstellen zur Bestimmung der Wasserspiegellagen ebenfalls von großer Bedeutung. Um die Unabhängigkeit zu gewährleisten, werden mehrere Messstellen sowohl im Ober- als auch im Unterstrombereich verwendet und daraus wird jeweils der Mittelwert gebildet.

\subsection{Netztest}

Die Verifikation des Einflusses der räumlichen Diskretisierung wird am geneigten Rechen mit der maximalen Neigung $\delta=30^{\circ}$ durchgeführt. Die numerische Simulation für diese Konfiguration erfolgte mit allen Netzen, vom gröbsten Netz N1 bis zum feinsten Netz N4. Als Vergleichswert dient dabei die Energieverlusthöhe $\Delta \mathrm{h}_{\mathrm{E}}$. Die Ergebnisse sind in Tab. 3 dargestellt:

Wie man der Tab. 3 entnehmen kann, weicht das Ergebnis für die Energieverlusthöhe $\Delta \mathrm{h}_{\mathrm{E}}$ beim gröbsten Netz um 2,06\% vom Ergebnis beim feinsten Netz N4 ab, welches hier als Referenzwert dient. Die Abweichung wird mit steigender Auflösung stetig kleiner und beträgt beim zweitfeinsten Netz N3 nur
Tab. 3 Auswertung Netztest am geneigten Rechen, $\delta=30^{\circ}$

\begin{tabular}{|l|l|l|}
\hline Netz [Nr.] & $\Delta \mathrm{h}_{\mathrm{E}}[\mathrm{mm}]$ & Abweichung zu N4 [\%] \\
\hline N1 & 22,23 & 2,06 \\
\hline N2 & 22,04 & 1,19 \\
\hline N3 & 21,91 & 0,59 \\
\hline N4 & 21,78 & - \\
\hline
\end{tabular}

noch 0,59\%. Das Ergebnis kann somit als netzunabhängig angenommen werden.

\subsection{Nullvariante}

Die besonderen Eigenschaften einer Baffle wurden eingangs schon erläutert. Darunter zählt auch das Merkmal, dass eine Baffle, die zwischen den Gitterlinien des Berechnungsnetzes definiert wird, für die Berechnung zur nächsten Gitterlinie verschoben wird. Basierend auf Voruntersuchungen mit einer undurchlässigen Baffle, ohne Zufluss und mit einer vorgegebenen Wasserspiegeldifferenz auf beiden Seiten, wird diese Adaptierung des Flächenobjektes untersucht. Für diese Nullvariante werden pro Rechenneigung jeweils zwei verschiedene Berechnungen mit dem gröbsten Netz N1 und dem feinsten Netz N4 durchgeführt um zu erkennen, welche Auswirkungen bei unterschiedlicher räumlicher Diskretisierung zu erwarten sind. Ausgewertet wird der sich ergebende Ruhewasserspiegel.

Beim geraden Rechen werden zwei verschiedene Positionierungsvarianten der Baffle untersucht. Bei der ersten Variante wurde die Baffle bei $\mathrm{x}=0,00 \mathrm{~m}$ positioniert, also in $\mathrm{x}$-Richtung gesehen immer zwischen den Zellen des Berechnungsnetzes. Bei der zweiten Variante wurde sie abhängig von der Netzauflösung, in x-Richtung immer genau in der Mitte der Zellen positioniert. Beim Netz N1 ergibt dies die Position $\mathrm{x}=0,05 \mathrm{~m}$ und beim Netz N4 die Position $\mathrm{x}=0,00625 \mathrm{~m}$ für die Baffle. Wie die Auswertung der Ergebnisse zeigt, wird die Baffle bei der zweiten Variante immer zur nächsten Zellgrenze hin verschoben, wodurch sich die beiden gewählten Varianten in der Berechnung und dem Ergebnis nicht voneinander unterscheiden. Zusätzlich dazu wurde noch eine dritte Variante mit einer um $\delta=30^{\circ}$ geneigten Baffle untersucht. Bei den geneigten Baffles erfolgt die Definition der Baffle wie erwartet in Form einer Treppe. Beispielhaft am Netz N4 ist dies in Abb. 4 zu erkennen. Basierend auf dieser Auswertung ist aber keine abrupte Änderung des Wasserspiegels zu 


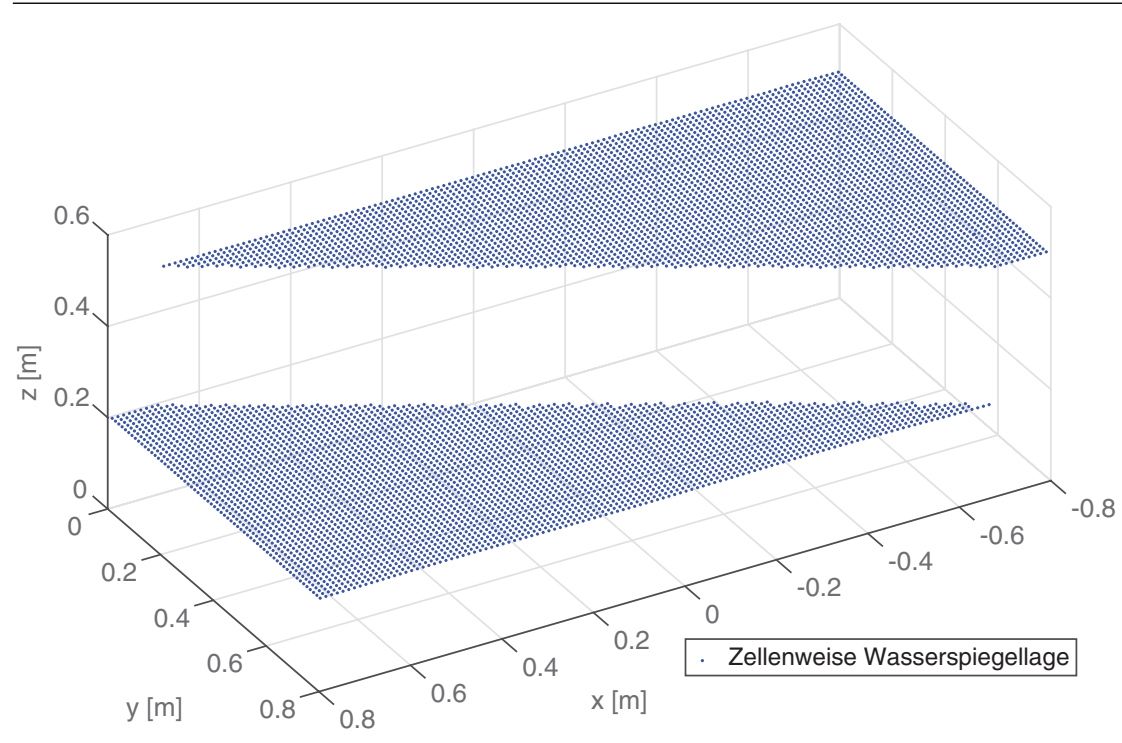

Abb. 5 Zellenweise Auswertung der Wasserspiegelhöhen ohne Interpolation mit MATLAB für die Nullvariante, geneigter Rechen, $\delta=30^{\circ}$, Netz N4

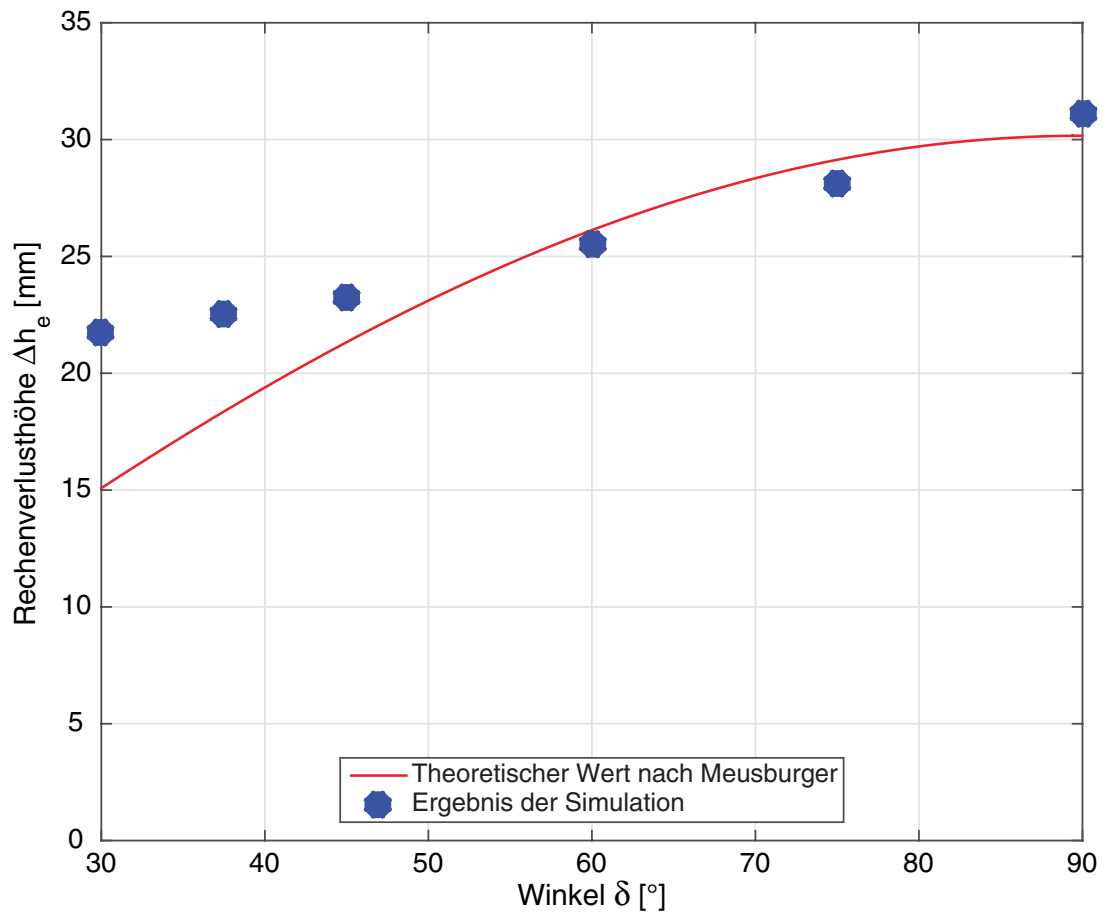

Abb. 6 Auswertung Einfluss der Rechenneigung für Netz N4

sehen. Das gleiche gilt auch für das grobe Netz N1, wobei für beide Netze keine Strömungsgeschwindigkeit auszumachen ist.

Um sicherzustellen, dass es sich um einen reinen Darstellungsfehler in der 3D-Ansicht handelt, wurden zusätzlich noch Längenschnitte in der $\mathrm{x}$-z-Ebene durch die Baffle ausgewertet. Dieses Verschmieren zeigte sich jedoch auch in
Tab. 4 Auswertung Einfluss der Rechenneigung für Netz N4

\begin{tabular}{|l|l|l|l|}
\hline$\delta\left[^{\circ}\right]$ & $\zeta_{Q}[-]$ & $\Delta \mathrm{h}_{E}[\mathrm{~mm}]$ & $\begin{array}{l}\text { Abweichung zum } \\
\text { theoretischen Wert } \\
\text { nach Meusburger } \\
(2002)[\%]\end{array}$ \\
\hline 90 & 1,790 & 31,11 & 2,45 \\
\hline 75 & 1,790 & 28,16 & 4,44 \\
\hline 60 & 1,790 & 25,57 & 4,52 \\
\hline 45 & 1,790 & 23,27 & 4,06 \\
\hline 37,5 & 1,790 & 22,54 & 15,39 \\
\hline 30 & 1,790 & 21,78 & 33,48 \\
\hline
\end{tabular}

wertung dieser Dateien in MATLAB zeigt die Abb. 5, wobei die Wasseroberfläche als Punktwolke ohne Interpolation dargestellt ist. Dabei ist sehr gut zu erkennen, dass die Software die beiden unterschiedlichen Wasserspiegellagen sehr wohl korrekt berechnet und die Baffle wie gewünscht einen Sprung zwischen den beiden Teilen erzeugt.

\section{Ergebnisse}

Sämtliche numerische Simulationen werden unabhängig von der Neigung des RechensmitdemgleichenEingabewertfür den quadratischen Verlustkoeffizienten $\zeta_{\mathrm{Q}}$ in FLOW-3D durchgeführt. Dieser Verlustbeiwert ergibt sich nach Meusburger (2002) $\mathrm{zu} \zeta_{\mathrm{Q}}=\zeta_{\mathrm{R}}=1,79$ [-]. Die Ergebnisse dieser Annahme werden in Abb. 6 den theoretischen Werten nach Meusburger (2002) entsprechend der Gl. 1 gegenübergestellt. Dabei werden die Ergebnisse des feinsten Netzes N4 bei verschiedenen horizontalen Anströmwinkeln $\delta$ mit dem jeweils durch den Korrekturfaktor k $\alpha$ abgeminderten Wert laut Meusburger (2002) verglichen.

Wie in der Abb. 6 zu erkennen ist, zeigt sich für den Bereich des Winkels $\delta=45$ bis $90^{\circ}$ (gerader Rechen) eine relativ gute Übereinstimmung der numerisch ermittelten Energieverlusthöhen mit den theoretisch ermittelten und abgeminderten Werten nach Meusburger (2002). In Tab. 4 ist die genaue Auswertung dieser Untersuchungen $\mathrm{zu}$ sehen. Bei einer weiteren Verringerung des Winkels $\delta$, was einer weiteren Verlängerung des Rechens entspricht, weichen die numerischen Ergebnisse doch deutlich vom theoretischen Wert nach Meusburger (2002) ab. Die 3DNumerik überschätzt dabei den Verlust mit dem gewählten Ansatz.

Als nächster Schritt werden die Ergebnisse sämtlicher numerischer 


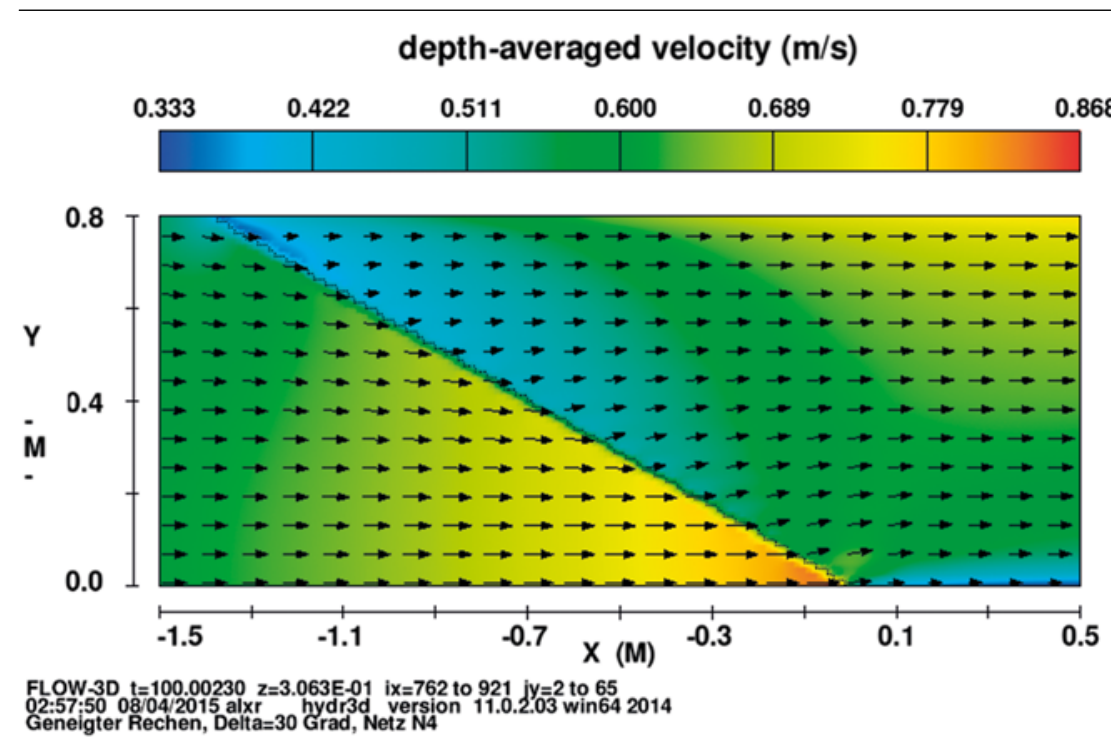

Abb. 7 Grundriss mit tiefengemittelten Geschwindigkeiten und Geschwindigkeitsvektoren, geneigter Rechen, $\delta=30^{\circ}$, Netz N4, $t=100 \mathrm{~s}$

Simulationen im Hinblick auf die Strömungszustände untersucht. Dafür werden unter anderem die tiefengemittelten Geschwindigkeiten im Grundriss analysiert und zusammen mit den Geschwindigkeitsvektoren im unmittelbaren Bereich vor und nach der Baffle ausgegeben. In Abb. 7 ist die exemplarische Auswertung für den Fall $\delta=30^{\circ}$ zu sehen. Rettemeier (2000) beschreibt aufgrund von Modellversuchen beim horizontal schräg angeströmten Rechen eine Veränderung der $\mathrm{Ge}$ schwindigkeitsverteilung im Grundriss. Grund dafür ist die keilförmige Verengung des Abflussquerschnittes, die mit fortlaufender Rechenlänge in Fließrichtung gesehen zu einer Erhöhung der
Fließgeschwindigkeiten vor dem Rechen führt. Dasselbe Bild zeigt sich auch bei den Ergebnissen der numerischen Simulationen in der vorliegenden Arbeit.

\section{Schlussfolgerungen und Ausblick}

Aus der vorliegenden Untersuchung lasst sich schließen, dass der vereinfachte Lösungsansatz mit der Baffle auch bei schräg gestellten Rechen prinzipiell verwendet werden kann, wobei dies jeweils für die projektspezifischen Gegebenheiten gesondert überprüft werden sollte. Bei den durchgeführten vereinfachten Untersuchungen konnte bei einem Anströmwinkel im Bereich $\delta=45$ bis $90^{\circ}$ mit der Baffle in FLOW-3D die Energieverlusthöhe entsprechend den theoretischen Werten nach Meusburger (2002) einfach integriert werden. Für Anströmwinkel $\delta<45^{\circ}$ ist mit einer Überschätzung zu rechnen. Es empfiehlt sich daher, die Verlusthöhen immer auf Plausibilität zu kontrollieren.

Die Ergebnisse der numerischen Simulationen wurden auch in Hinblick auf die Strömungszustände untersucht. Strömungsphänomene am einzelnen Rechenstab können mit einer Baffle nicht abgebildet werden. Die Strömungsverhältnisse in der Fläche decken sich jedoch mit den Beobachtungen an Modellversuchen in der Literatur. Die Messungen für den untersuchten Vorversuch zum Seilrechen sind Teil der aktuellen Forschung.

FLOW-3D übernimmt somit bei der numerischen Simulation selbstständig die Berücksichtigung der Schrägstellung der Baffle zur Hauptströmungsrichtung hin. Als Eingabewert in FLOW-3D für den quadratischen Verlustkoeffizient $\zeta_{Q}$ der Baffle muss einfach nur der theoretische Verlustbeiwert $\zeta_{R}$ des geraden Rechens nach Meusburger (2002) eingesetzt werden. Die Anwendbarkeit der Baffle als vereinfachte Implementierung von Rechenverlusten ist somit auch bei schräg angeordneter Rechenebene mit der entsprechenden Kontrolle möglich.

Open Access Dieser Artikel unterliegt den Bedingungen der Creative Commons Attribution License. Dadurch sind die Nutzung, Verteilung und Reproduktion erlaubt, sofern der/die Originalautor/en und die Quelle angegeben sind.

\section{Literatur}

Aufleger, M. (2014): Der Seilrechen als Fischschutz- und Fischleiteinrichtung an Laufwasserkraftanlagen. Antragsteller: Universität Innsbruck, Arbeitsbereich Wasserbau, Institut für Infrastruktur. Projektpartner: Universität Innsbruck, Arbeitsbereich für Angewandte Mechanik, Institut für Grundlagen der Technischen Wissenschaften; FFBOKU Wien, Institut für Hydrobiologie und Gewässermanagement; Albatros Engineering $\mathrm{GmbH}$

Aufleger, M., Brinkmeier, B. (2015): Wasserkraftanlagen mit niedrigen Fallhöhen - Verschiedene Konzepte im kritischen Vergleich. In: Österreichische Wasser- und Abfallwirtschaft 67/7-8. doi:10.1007/s00506-015-0247-6

Böttcher, H., Brinkmeier, B., Aufleger, M. (2014): Der Seilrechen als neues Fischschutzkonzept - Untersuchung der technischen Machbarkeit. In: VAW Mitteilungen Nr. 227, ETH Zürich. http://people.ee.ethz.ch/vawweb/vaw_mitteilungen/227/227.pdf
Chatellier, L., Wang, R.W., David, L., Courret, D., Larinier, M. (2011): Experimental characterization of the flow across fish-friendly angled trashrack models. In: Proceedings of the 34th World Congress of the International Association for Hydro- Environment Research and Engineering: 33rd Hydrology and Water Resources Symposium and 10th Conference on Hydraulics in Water Engineering, Engineers Australia.

Cuchet, M. (2013): Laboruntersuchungen des Wanderverhaltens an geneigten Rechen zu Fischschutz und Fischabstieg. In: Wasserbau und Wasserwirtschaft: Berichte des Lehrstuhls und der Versuchsanstalt für Wasserbau und Wasserwirtschaft Technische Universität München $\mathrm{Nr}$. 128. http://permalink.obvsg.at/AC07022994 Flow Science, Inc (2014): FLOW-3D Version 11.0.3 - User manual
Gabl, R., Gems, B., De Cesare, G., Aufleger, M. (2014): Anregungen zur Qualitätssicherung in der 3-D-numerischen Modellierung mit FLOW-3D (Contribution to quality standards for 3D-numerical simulations with FLOW-3D). In: WasserWirtschaft - Fachzeitschrift für Wasser und Umwelttechnik 03/2014. doi:10.1365/ s35147-014-0938-0

Kirschmer, O. (1926): Untersuchungen über den Verlust an Rechen. In: Mitteilungen des Hydraulischen Instituts der Technischen Hochschule München Nr. 1. http://permalink.obvsg.at/ AC02901281

Kriewitz, C.R. (2015): Leitrechen an Fischabstiegsanlagen: Hydraulik und fischbiologische Effizienz. VAW Mitteilungen Nr. 230, ETH Zürich. http://e-collection.library.ethz.ch/eserv/ eth:47523/eth-47523-02.pdf

Krzyzagorski, S. (2015): Numerische Berechnung eines schräg angeströmten Rechens. Masterarbeit, Betreuer: Univ.-Prof. Dr.-Ing. habil. Markus Aufleger, Universität Innsbruck, Arbeitsbereich für Wasserbau 
Raynal, S., Courret, D., Chatellier, L., Larinier, M., David, L. (2013): An experimental study on fishfriendly trashracks Part 2 Angled trashracks. In: Journal of Hydraulic Research Volume 51 Issue 1. doi:10.1080/00221686.2012.753647

Meusburger, H. (2002): Energieverluste an Einlaufrechen von Flusskraftwerken. Dissertation. VAW Mitteilungen Nr. 179, ETH Zürich. http:// people.ee.ethz.ch/ vawweb/vaw_mitteilungen/ 179/179_g.pdf

Mosonyi, E. (1966): Wasserkraftwerke, Band 1 Niederdruckanlagen. VDI-Verlag Düsseldorf. http://permalink.obvsg.at/AC00064624
Rettemeier, K. (2000): Laufwasserkraftwerke und der Schutz wandernder Fische Lösungswege zu einer Koexistenz. Ein Bericht der Aalschutzinitiative Rheinland Pfalz/RWE Energie AG (Teil 2). In: Mitteilungen des Lehrstuhls und Instituts für Wasserbau und Wasserwirtschaft der Rheinisch-Westfälischen Technischen Hochschule Band 121. http://permalink.obvsg.at/ AC02524156

Ritsch, S. (2015): Hydraulische Untersuchungen an Schrägrechen zum Fischschutz, Masterarbeit, Betreuer: Univ.-Prof. Dr.-Ing. habil. Markus Aufleger, Universität Innsbruck, Arbeitsbereich für Wasserbau
Waldy, M., Gabl, R., Seibl, J., Aufleger, M. (2015): Alternative Methoden für die Implementierung von Rechenverlusten in die 3D-numerische Berechnung mit FLOW-3D (Alternative methods for the implementation of trash rack losses in the 3D-numerical calculation with FLOW-3D). In: Österreichische Wasser- und Abfallwirtschaft 67/12. doi:10.1007/s00506-014-0205-8

Zimmermann, J. (1969): Widerstand schräg angeströmter Rechengitter. In: Universität Fridericana Karlsruhe, Theodor-RehbockFlußbaulaboratorium Mitteilungen, Heft Nr. 157. http://permalink.obvsg.at/AC00235212 